\title{
Enhanced Edge Detection Technique for SAR Images
}

\author{
P. Surya Prasad \\ Associate professor \\ MVGR College of Engineering \\ Vizianagaram, \\ Andhra Pradesh, India
}

\author{
B. Prabhakara Rao, Ph.D \\ Professor \\ JNTUK, Kakinada \\ East Godavari Dist \\ Andhra Pradesh, India
}

\author{
PNRL Chandrasekhar \\ Associate professor \\ GITAM College of Engg \\ Visakhapatnam \\ Andhra Pradesh, India
}

\begin{abstract}
In this work a novel technique for automatic edge enhancement and detection in synthetic aperture radar (SAR) images is proposed. The edge enhancement phase has been proven to be critical in heterogeneous SAR images, and the method proposed is a good solution that can be used to deal with this type of data. The method proposes a robust edge enhancement directly in the wavelet transformed domain based on a combination of wavelet coefficients at different scales. It does not require any type of pre filtering of input data, and is independent of the statistics of the input image. The adaptation capability of the method to very diverse scenarios with no need of a priori knowledge or settings is a useful feature in view of its integration in an unsupervised segmentation.
\end{abstract}

\section{General Terms}

Image Processing.

\section{Keywords}

Edge detection, synthetic aperture radar (SAR), stationary wavelet transform.

\section{INTRODUCTION}

Synthetic Aperture Radar (SAR) is used to obtain highresolution images from broad areas of terrain. It has been used to estimate features like the dampness of soil, the thickness of the forest, or the roughness of the sea. It is capable of operating under inclement weather conditions, day or night These images provide high-resolution capability, making them helpful for the global observation of the Earth for environmental and security issues.. Processing of SAR data is required to extract relevant features, such as objects or buildings. Detection of such objects is based on the detection of locally bright pixels, followed by clustering of neighborhoods of pixels. Synthetic-Aperture Radar (SAR) is a form of radar whose defining characteristic is its use of relative motion, between an antenna and its target region, to provide distinctive long-term coherent-signal variations that are exploited to obtain finer spatial resolution than is possible with conventional beam-scanning means. SAR is usually implemented by mounting, on a moving platform such as an aircraft or spacecraft, a single beam-forming antenna from which a target scene is repeatedly illuminated with pulses of radio waves at wavelengths anywhere from a meter down to millimeters. The many echo waveforms received successively at the different antenna positions are coherently detected and stored and then post-processed together to resolve elements in an image of the target region. As SAR is a kind of coherence radar system, its image is generally degraded by speckle. The presence of speckle limits the usage of SAR image. Therefore, speckle reduction is a fundamental and essential process used in SAR image analysis.

\section{EDGE DETECTION METHODS}

The estimation of the boundary location of an object is an important subject in image processing and edge detection is a fundamental issue in SAR image analysis. Since edge detection has been studied, several techniques for edge enhancement are proposed. Robust edge detection techniques are essentially based on the following two steps: edge enhancement and decision. Unlike optical images, in SAR data, which is highly heterogeneous, a robust edge enhancement phase is critical in providing acceptable detection rates. This phase is usually performed through techniques that are related to derivation, namely, simple differences, Sobel filter [1], Roberts filter, Prewitt filter [2], morphological gradients etc., possibly combined with smoothing. However, there are three problems by the use of derivative as edge detector: i) false edges produced by noise are enhanced; ii) ramp edges produce lower derivative magnitude; iii) the location of the ramp edges are not accurate. Several edge detection methods as proposed by Marr-Hildreth [3], Canny [4] and Perona-Malik [5] provide tools to overcome the edge detection problems by applying the derivative to edge detection. Most of these methods apply a smooth gaussian operator in order to suppress the noisy edges and then estimate the location of the edges by detection of the zero-crossing on the second derivative of an image. Edge detectors proposed by Petrou-Kitter and Zang et. al. are based on ramp edges model. Petrou-Kitter [6] propose a ramp edge model to an optimal edge detector. D. Wang $[7,8]$ proposed a multiscale gradient algorithm that enhances the morphological gradient of ramp edges however the edge of thin objects can be lost. Due to existence of speckle, edge detection in SAR images is a very challenging task and the above methods provide limited efficiency in SAR applications due to the presence of a speckle which is a multiplicative noiselike pattern [9]. This paper aims at presenting a novel method for edge enhancement in SAR images based on the exploitation of the information provided by the wavelet coefficients. The usual edge detectors, successful with incoherent images, yield poor results when applied to radar images, especially those with a small number of looks. Their performance de- grades seriously because they are very sensitive to the speckle noise.

In the past, several multiscale approaches, which depend on the analysis of the information contained in the wavelet domain, were proposed [10]-[13]. The method proposed in this paper is based on a different way of manipulating multiscale data [14]. It does not assume any statistical distribution of the input data and any particular type of edge. It works exclusively in the transformed domain. Several algorithms are based on the wavelet theory like the study in [14] or [15] proposes an edge detector based on a threshold 
operation of wavelet coefficients. Despite a low computational cost and a good contrast, detected edges are too thick. The approach proposed in this paper will tackle at the same time the robustness and the precision issues of edge enhancement.

\section{PROPOSED METHOD}

A novel method for edge enhancement in SAR images which uses stationary wavelet, $\log$ transforms and works for any type of edge is proposed. The method uses Haar wavelet transform for multiscale analysis.

\subsection{Stationary wavelet transform}

Unlike the discrete wavelet transform, DWT [8], which downsamples the approximation coefficients and detail coefficients at each decomposition level, the SWT (stationary wavelet transform) does not incorporate the downsampling operations. Thus, the approximation coefficients and detail coefficients at each level are the same length as the original signal. The SWT upsamples the coefficients of lowpass and highpass filters at each level. The upsampling operation is equivalent to dilating wavelets. With increasing levels of decomposition, the resolution of SWT coefficients decreases.

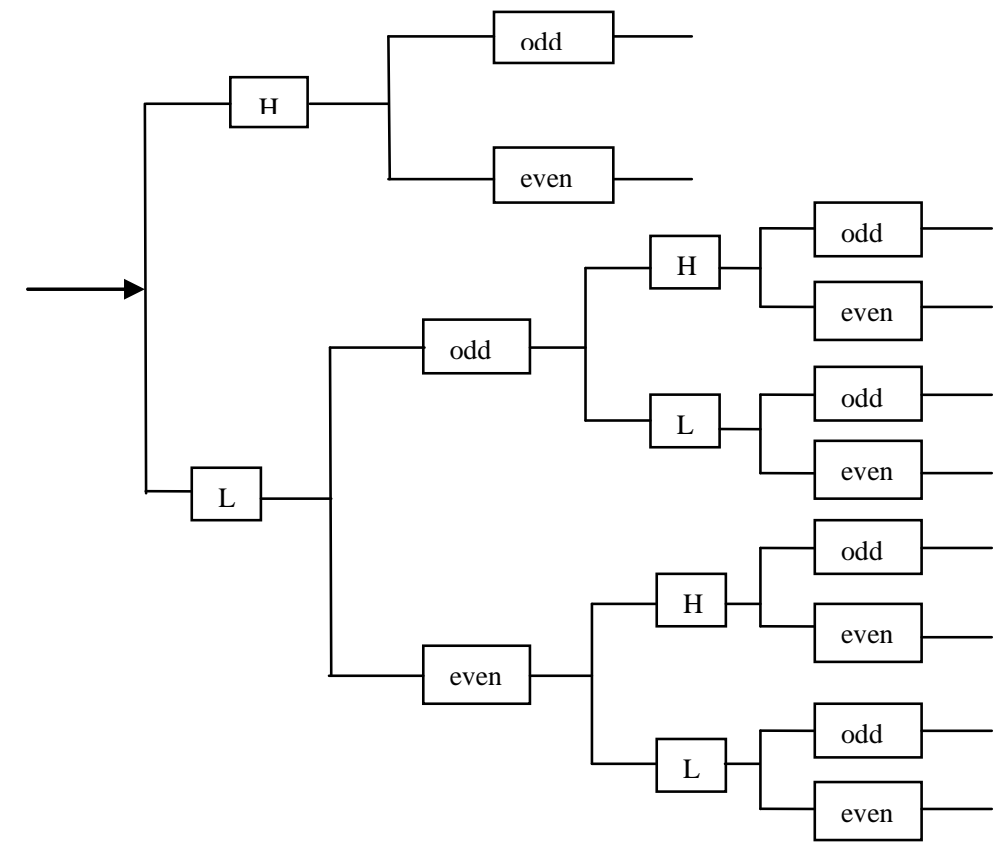

Fig.1 Stationary Wavelet Transform

The stationary discrete wavelet transform was independently used several times, for different purposes and with different names, e.g. shift/translation invariant wavelet transform, stationary wavelet transform, or redundant wavelet transform. The key point is that it is redundant, shift invariant, and gives a denser approximation to the continuous wavelet transform than the approximation provided by the orthonormal discrete wavelet transform (DWT). From the filter bank point of view, both even and odd downsamples are kept, and lowpass bands are further split. The structure of a two level stationary wavelet transform and its inverse stationary wavelet transform are shown in Figure 1and Figure 2.

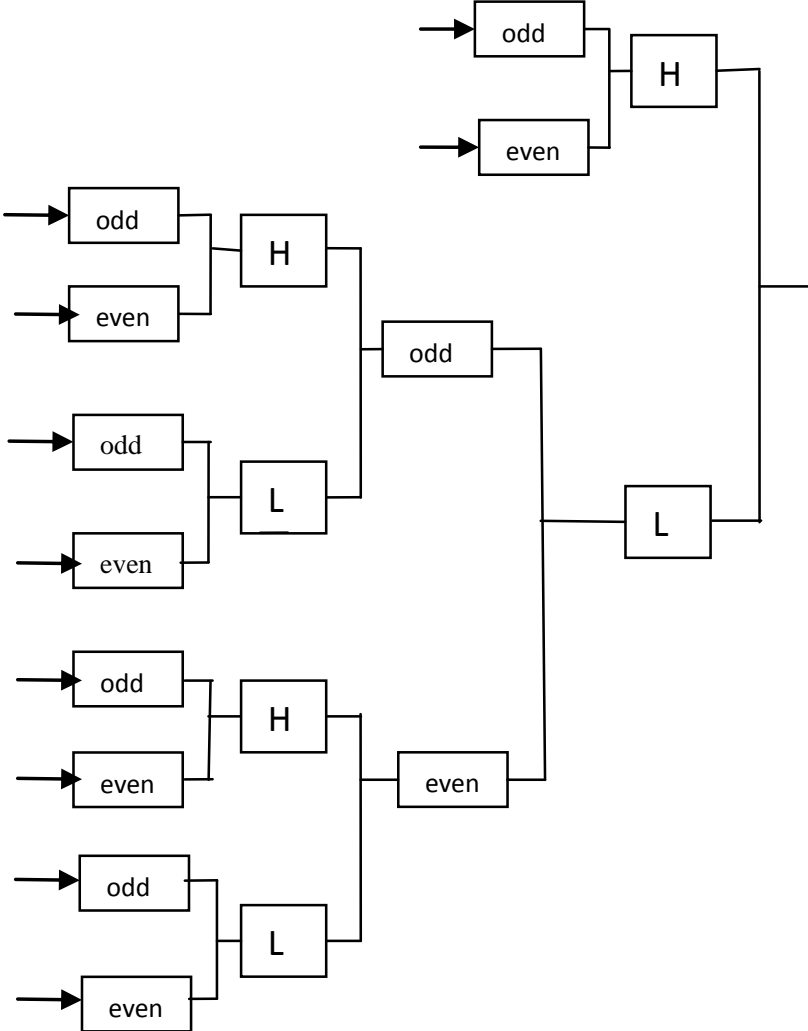

Fig. 2 Two level Inverse Stationary Wavelet Transform

\section{PROCESSING}

The algorithm for edge enhancement in SAR images, which is proposed in this project, relies on the difference of behavior along the wavelet scales of the speckle in front of the edges. On the one hand, the discontinuities are highlighted by the wavelet transform, and they tend to persist over scales. On the other hand, the speckle [9] is progressively smoothened and moreover, it is almost spatially uncorrelated between scales. The flowchart of the proposed algorithm is shown in Figure 3

\subsection{Logarithm}

In the first step the logarithm transformation is applied to the original signal in order to make the multiplicative speckle to Gaussian additive and signal independent. This operation is also helpful to reduce the large dynamic range of SAR data.

\subsection{SWT}

After the logarithmic operation, SWT in two dimensions is applied from which three bandpass components are obtained, where each one enhances the discontinuities in a different direction. After normalizing each of the above subbands to their maximum and then taking the absolute values, the pointwise maximum across each of the three subbands is evaluated.

\subsection{Normalization}

A correct implementation of the algorithm proposed must also deal with situations where a subband does not contain a vessel, as the proposed normalization may lead to noise amplification. To deal with this effect, an improved normalization step is implemented which performs first an evaluation of the relative level of the noise floor with respect to the maximum in each wavelet subband. If a feature (i.e., a vessel) is present in a particular subband, the histogram shall be heavy tailed, and the normalization is performed as usual using the maximum of the corresponding subband. If not, the 
normalization factor is a number of times (usually three) the standard deviation in order to ensure low values on this subband [14]. Since, the components in the same scale are uncorrelated, taking the maximum value pixel per pixel permits avoiding as much as possible the speckle. The same operation is carried out with different scales. Then, the different intermediate maxima previously calculated are combined through pointwise multiplication.

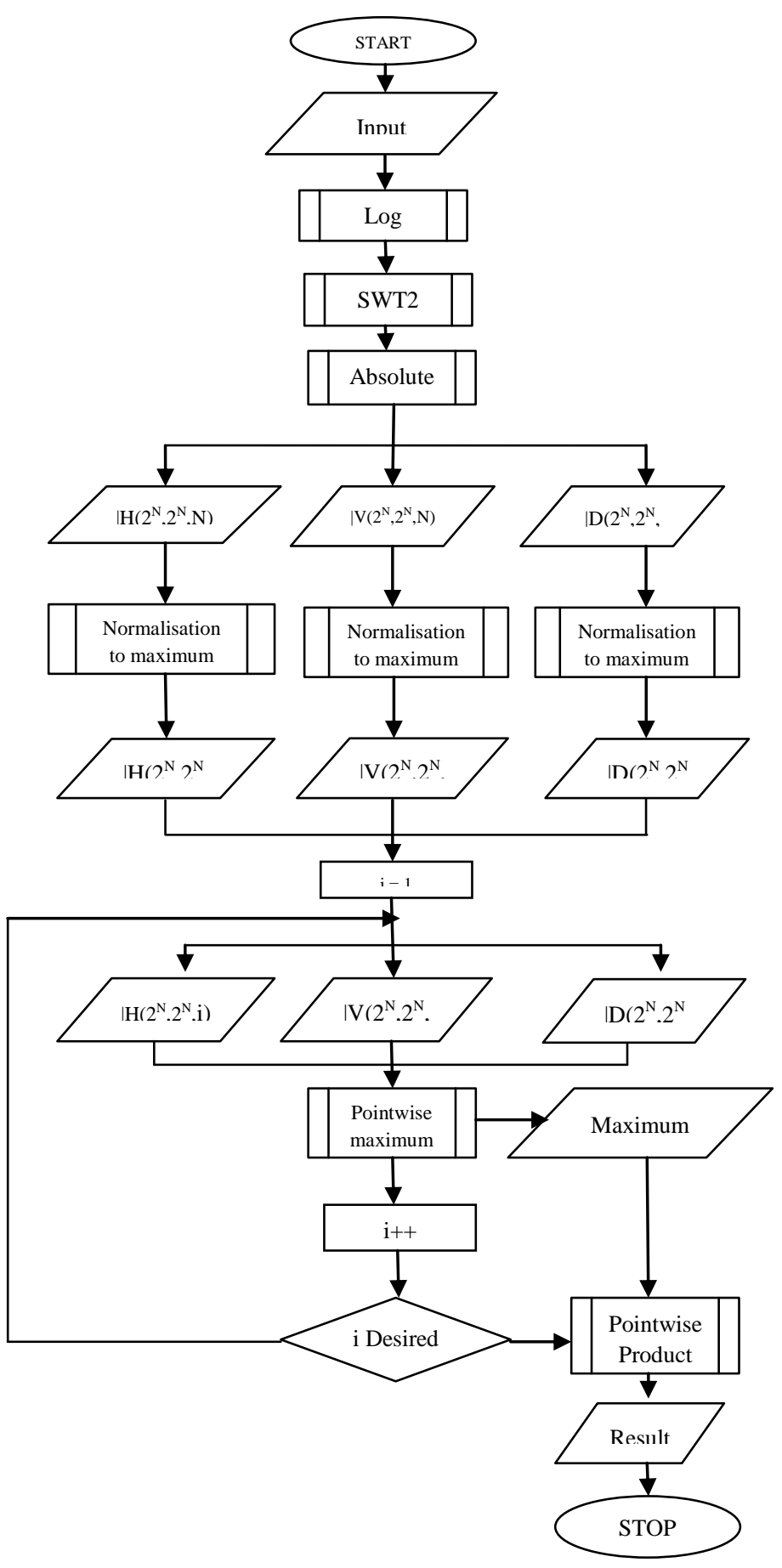

Fig. 3 Flowchart of the proposed algorithm

\subsection{Pointwise product}

For each pixel, the output value corresponds to the product of the values for that pixel of the intermediate maxima matrices obtained in the previous step. If necessary and if some kind of a priori information is available, the number of iterations can be adjusted accordingly. Otherwise, in common situations five iterations are sufficient to provide satisfactory results regarding the usual range of the resolution of SAR images.

\subsection{Important properties}

The proposed algorithm has an prominent property of simplicity. The computational cost is low and it is an iterative process that requires two operations per iteration: application of a single iteration of the SWT and the evaluation of the pointwise maxima. No prefiltering step is added, and it doesn't depend on the statistics of the input image. As the algorithm provides a result directly in the wavelet domain, as a consequence, contrary to conventional filters, it does not require any inversion step such as in [10], which is usually an awkward process, often introducing artifacts when wavelet coefficients are processed. Working on the transformed domain implies considering differential values rather than absolute ones. The result of the proposed technique is the contrast achieved between edges and background, which is the main objective of any edge enhancement algorithm. The proposed method can be employed for automatic coastline detection in SAR images and automatic ship detection.

\section{SIMULATION RESULTS}

Performing an automatic extraction of the coastline from remote-sensing data is meaningful because sometimes it is challenging to obtain a precise map of the coastline in particular regions and circumstances with other conventional tools. Moreover, the coastline is subject to a erosion: natural, abrupt due to environmental disaster such as a tsunami, and artificial due to a man-made alteration of the coastline. The monitoring of the coast provides needed information about the behavior of the ocean currents. A set of SAR images has been taken into consideration for validation purposes and have been tested. The results obtained for the proposed edge enhancement algorithm for a particular SAR image is compared with the results of the application of Canny, Sobel, LoG, Roberts and Prewits algorithms. The observation of the results obtained in SAR images confirms a good match of the detected coastline to the shoreline that is visually inferred. Moreover, the correct performance of the method is also observed despite the diversity of scenarios.

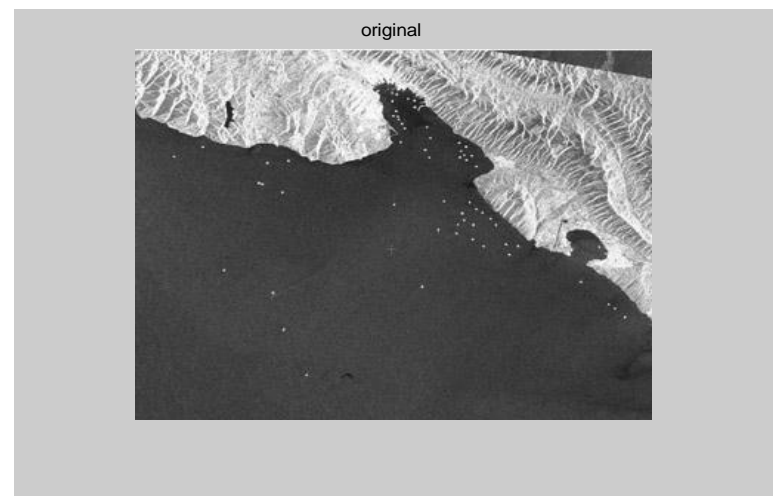

Fig. 4 Original coastline image 


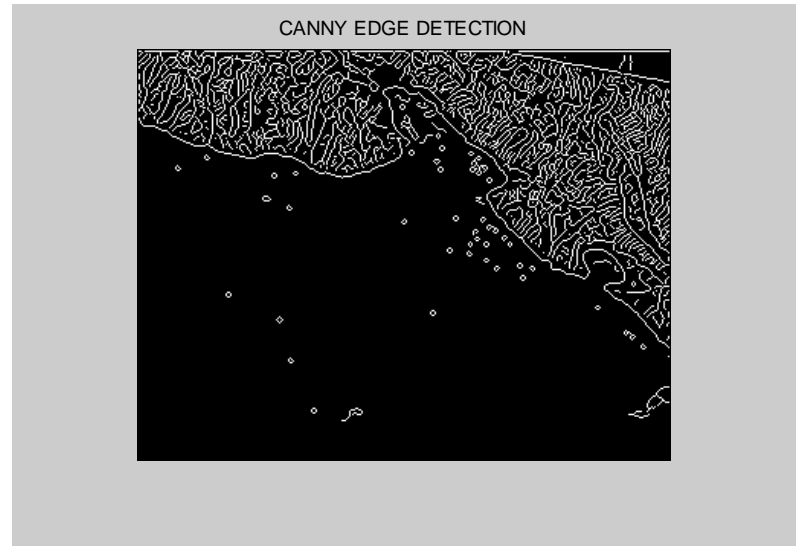

Fig. 5 Result of the application of Canny Edge Detection

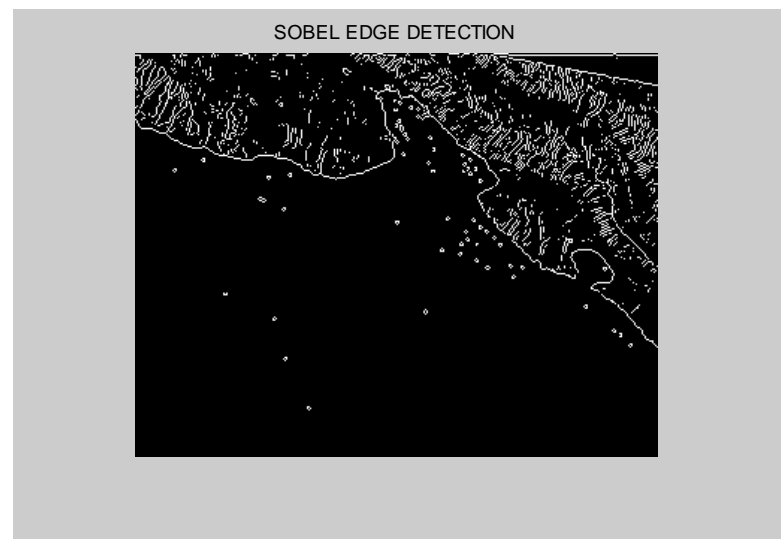

Fig. 6 Result of the application of Sobel Edge Detection

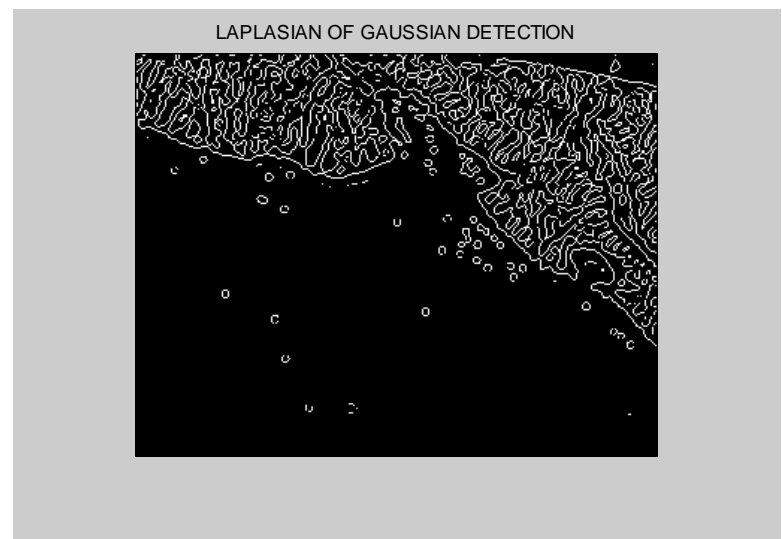

Fig. 7 Result of the application of Laplacian of Gaussian

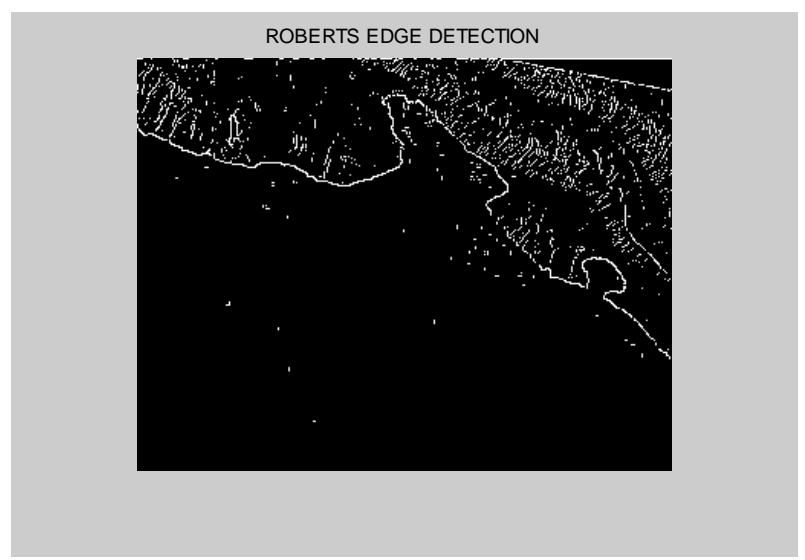

Fig. 8 Result of the application of Roberts Edge Detection

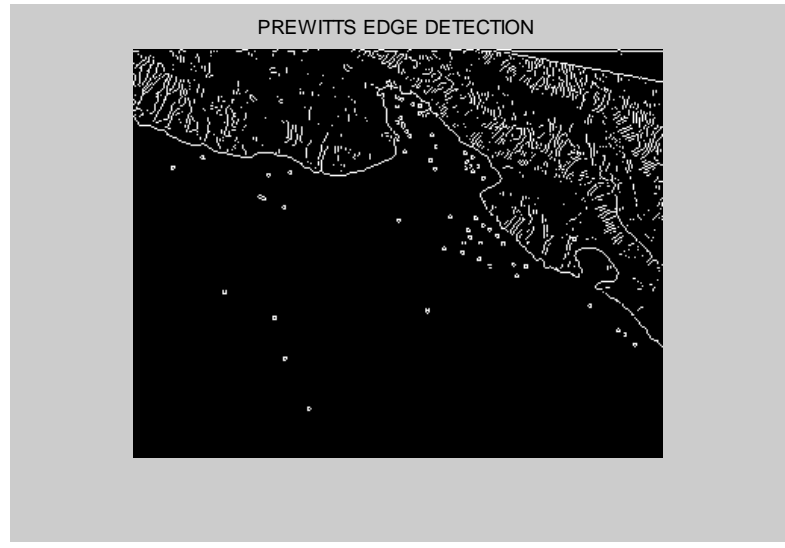

Fig. 9 Result of the Prewits Edge Detection

\subsection{Result of the proposed algorithm}

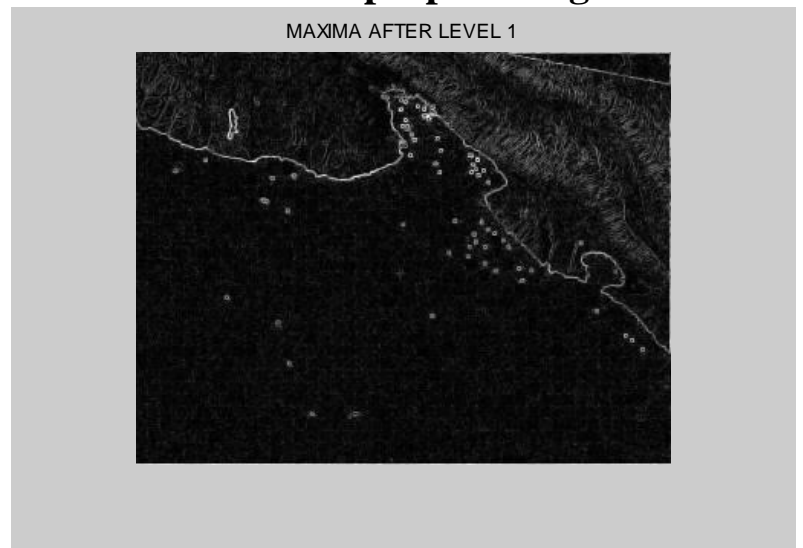

Fig. 10 Maxima after level 1 decomposition

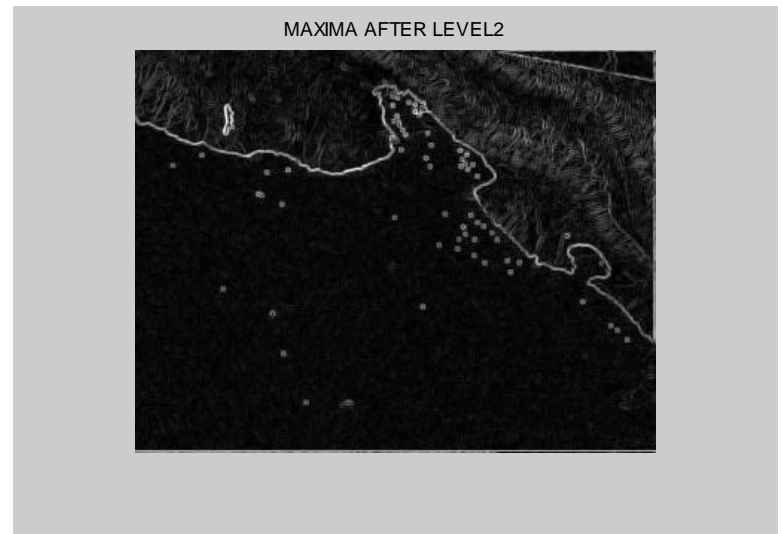

Fig. 11 Maxima after level 2 decomposition

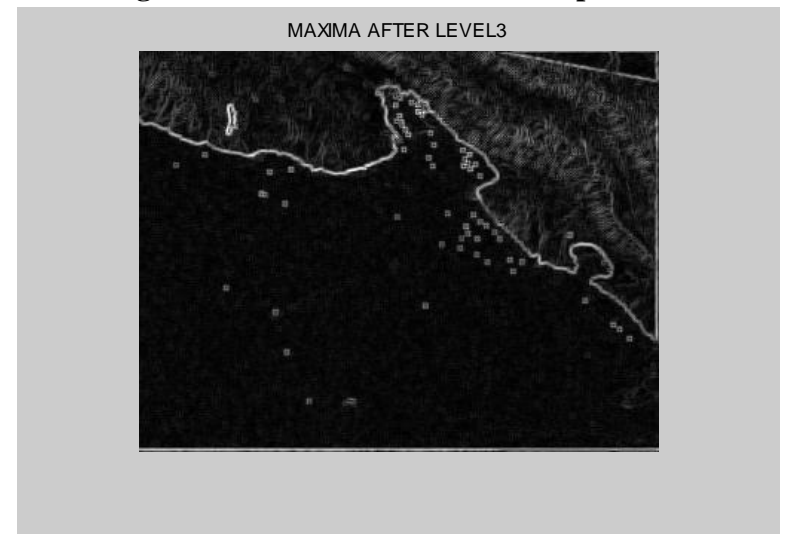

Fig. 12 Maxima after level 3 decomposition 


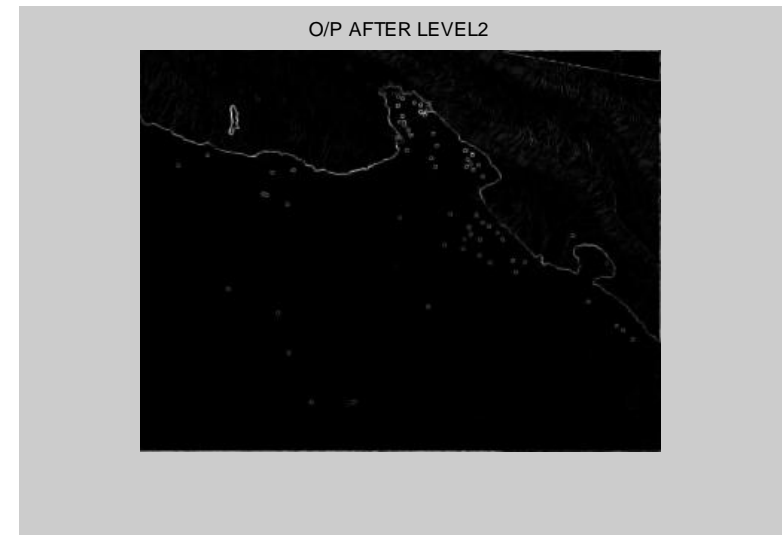

Fig. 11 Output after level 2 decomposition

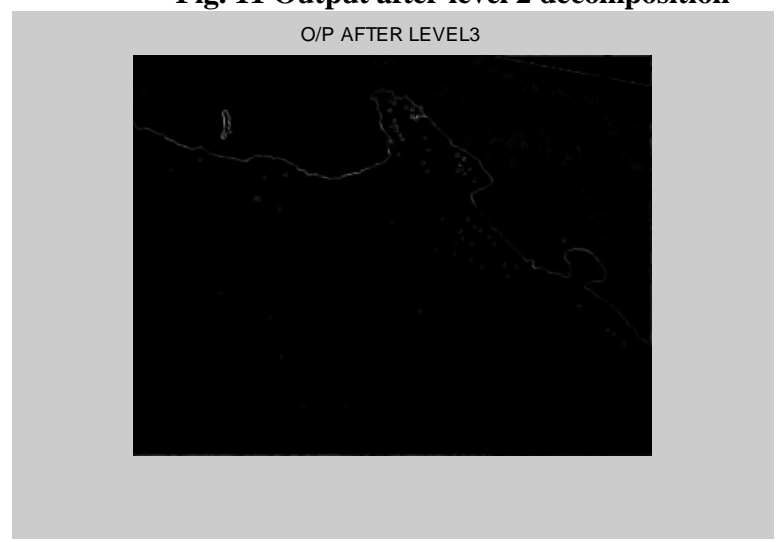

Fig. 11 Output after level 3 decomposition

\section{CONCLUSIONS}

The method proposes a robust edge enhancement directly in the wavelet transformed domain, The edge enhancement phase has been proved to be critical in heterogeneous SAR images, and the method proposed in this paper constitutes a good solution which can be used to deal with this type of data. It does not require any type of prefiltering of data, and it is independent of the statistics of the input image. The adaptation capability of the method to very diverse scenarios with no need of a priori knowledge or settings is a useful feature in view of its integration in an unsupervised segmentation.

\section{REFERENCES}

[1] W. K. Pratt, Digital Image Processing. New York: Wiley, 1978.

[2] J.M. S. Prewitt, "Object enhancement and extraction," in Picture Processing and Psychopictorics, B. S. Lipkin and A. Rosenfeld, Eds. New York: Academic, 1970, pp. 75-149.
[3] Marr, D. and Hildreth, E. C., Theory of Edge Detection, Proc. of the Royal Society of London B207, pp. 187-217, 1980.

[4] Canny, J., A Computational Approach to Edge Detection, PAMI,V.8,No. 6,pp. 679-698, 1986.

[5] Perona, P. and Malik, J., Scale-Space and Edge Detection Using Anisotropic Diffusion, PAMI ,V.12, No. 7, 1990.

[6] Petrou, M. and Kitter, J., Optimal Edge Detectors for Ramp Edges, PAMI, V. 13, No. 5, pp. 483-491, 1991.

[7] Wang, D., A Multiscale Gradient Algorithm for Image Segmentation using Watersheds,Pattern Recognition, V. 30, No. 12, pp. 2043-2052, 1997.

[8] Wang, Z., Rao, K. R. and Ben-Arie, J., Optimal Ramp Edge Detection Using Expansion Matching, PAMI, V. 18, No. 11, pp. 1092-1097, 1996.

[9] J. W. Goodman, Speckle Phenomena in Optics: Theory and Applications. Englewood, CO: Roberts \& Company, 2007.

[10] Y. Xu, J. B. Weaver, D. M. Healy, and J. Lu, "Wavelet transform domain filters: A spatially selective noise filtration technique," IEEE Trans. Image Process., vol. 3, no. 6, pp. 747-758, Nov. 1994.

[11] B. M. Sadler and A. Swami, "Analysis of multiscale products for step detection and estimation," IEEE Trans. Inf. Theory, vol. 45, no. 3, pp. 1043-1051, Apr. 1999.

[12] G. Cuartero, V. Meas-Yedid, V. Galy, U. Nehrbass, and J.-C. Olivo-Marine, "Three dimensional spot detection by multiscale analysis," in Proc. Int. Conf. Image Process., Oct. 7-10, 2001, vol. 1, pp. 317-320.

[13] J. Kuo and K.-S. Chen, "The application of wavelets correlator for ship wake detection in SAR images," IEEE Trans. Geosci. Remote Sens, vol. 41, no. 6, pt. 2, pp. 1506-1511, Jun. 2003

[14] Mariví Tello Alonso, Carlos López-Martínez, Jordi J. Mallorquí, and Philippe Salembier, "Edge Enhancement Algorithm Based on the Wavelet Transform for Automatic Edge Detection in SAR Images", IEEE transactions on geoscience and remote sensing, vol. 49, no. 1, January 2011.

[15] E. Romaneessen, A. Niedermeier, N. Winkel, and S. Lehner, "Improved bottom topography in the Elbe estuary using wavelet and active contour methods on SAR images," in Proc. IGARSS, Jul. 1999, vol. 3, pp. 1674-1676.

[16] S. Madchakham, P. Thitimajshima, and Y. Ragsanseri, "Edge detection in speckled SAR images using wavelet decomposition," in Proc. ACRS, Nov. 2001, vol. 2, pp. $1307-1310$. 